\title{
Tratamiento de la atelectasia lobar aguda mediante insuflación selectiva transbroncoscópica en pacientes graves intubados: experiencia en un hospital general
}

Treatment of acute lobar atelectasis by selective trans-bronchoscopic insufflation

in critically ill intubated patients: a general hospital experience

Tratamento da atelectasia lobar aguda por insuflação transbroncoscópica seletiva

em pacientes intubados graves: experiência em um Hospital Geral

René Agustín Flores-Franco, ${ }^{\star}$ Alexandro Franco-Estrada, ${ }^{*}$ Adrián Velázquez-Jáuregui*

\section{RESUMEN}

Introducción: La atelectasia lobar aguda (ALA) es una complicación frecuente en pacientes bajo apoyo ventilatorio mecánico que suele resolverse con ayuda del aspirado de secreciones traqueobronquiales y fisioterapia respiratoria. No obstante, existen casos refractarios a dicho tratamiento en los cuales la broncoscopia es de gran utilidad. La insuflación transbroncoscópica del lóbulo atelectasiado ha sido descrita, pero su técnica e indicaciones aún no han sido consensados en la literatura. Exponemos nuestra experiencia con una técnica personalizada.

Material y métodos: Revisión retrospectiva de ocho casos ilustrativos de ALA de más de cinco días de evolución, refractaria a tratamiento conservador según nuestro protocolo local institucional, tratados mediante insuflación transbroncoscópica con bolsa de Ambu.

Resultados: De los ocho casos, observamos cuatro resoluciones inmediatas, tres tardías (24 horas después) y uno que cursó con complicaciones.

Conclusiones: Con el debido entrenamiento, la insuflación transbroncoscópica puede ser una opción útil de tratamiento en pacientes con ALA bajo apoyo ventilatorio mecánico.

Palabras clave: Atelectasia pulmonar, ventilación mecánica, broncoscopia, fisioterapia respiratoria.

\section{ABSTRACT}

Introduction: Acute lobar atelectasis $(A L A)$ is a frequent complication in patients under mechanical ventilatory support that is usually resolved with the help of suctioning of tracheobronchial secretions and with respiratory physiotherapy. However, there are refractory cases in which bronchoscopy would be useful. Trans-bronchoscopic insufflation of the atelectatic lobe has been described but its technique and indications have not yet subject to consensus. We expose our experience with a personalized technique of trans-bronchoscopic insufflation in intubated patients with ALA

Material and methods: Retrospective review of eight illustrative cases of ALA with more than five days of evolution and refractory to conservative treatment according to our local institutional protocols, treated by trans-bronchoscopic insufflation with an Ambu bag.

Results: Of the eight cases, we observe four cases with immediate resolution of $A L A$, three with a late resolution (24 hours later) and one with a complicated result. Conclusions: With the proper training, trans-bronchoscopic insufflation may be a useful treatment option in patients with ALA under mechanical ventilatory support. Keywords: Pulmonary atelectasis, mechanical ventilation, bronchoscopy, physiotherapy.

\section{RESUMO}

Introdução: $A$ atelectasia lobar aguda (ALA) é uma complicação comum em pacientes em suporte ventilatório mecânico que geralmente se resolve com o auxílio de aspiração de secreções traqueobrônquicas e fisioterapia respiratória. Porém, há casos refratários ao referido tratamento em que a broncoscopia é muito útil. A insuflação transbroncoscópica do lobo da atelectasia foi descrita,

\footnotetext{
* Hospital General Regional «Dr. Salvador Zubirán Anchondo». Chihuahua, Chih., México

Recibido: 22/12/2019. Aceptado: 21/01/2020.

Citar como: Flores-Franco RA, Franco-Estrada A, Velázquez-Jáuregui A. Tratamiento de la atelectasia lobar aguda mediante insuflación selectiva transbroncoscópica en pacientes graves intubados: experiencia en un hospital general. Med Crit. 2021;35(1):28-33. https://dx.doi.org/10.35366/99151
}

www.medigraphic.com/medicinacritica mas sua técnica e indicações ainda não foram acordadas na literatura. Expomos nossa experiência com uma técnica personalizada.

Material e métodos: Revisão retrospectiva de 8 casos ilustrativos de ALA com mais de 5 dias de evolução, refratários ao tratamento conservador de acordo com nosso protocolo institucional local, tratados por insuflação transbroncoscópica com bolsa Ambu.

Resultados: Dos 8 casos, observamos 4 resoluções imediatas, três tardias (24 horas depois) e uma que apresentou complicações.

Conclusões: Com o treinamento adequado, a insuflação transbroncoscópica pode ser uma opção de tratamento útil em pacientes com ALA em suporte ventilatório mecânico.

Palavras-chave: Atelectasia pulmonar, ventilação mecânica, broncoscopia, fisioterapia respiratória.

\section{INTRODUCCIÓN}

La atelectasia lobar aguda (ALA) es el colapso de los segmentos principales del pulmón que ocurre como una complicación frecuente en pacientes intubados y bajo apoyo ventilatorio mecánico de la Unidad de Cuidados Intensivos ( $\mathrm{UCI})$, sobre todo cuando existen condiciones predisponentes como edad avanzada, obesidad, exceso de secreciones en las vías respiratorias, debilidad de la musculatura respiratoria o edema pulmonar. ${ }^{1}$ El principal mecanismo de producción de la ALA es la obstrucción de la vía aérea por un tapón de moco, resultado de un incremento en las secreciones asociado con la disminución del aclaramiento mucociliar epitelial normal. Además, el oxígeno suplementario promueve la absorción y colapso alveolar por un fenómeno de desnitrogenización, así como la inactivación o pérdida del surfactante alveolar. Las consecuencias fisiológicas de la ALA incluyen la alteración en la distensibilidad pulmonar, hipoxemia arterial y el aumento de carga de trabajo ventilatorio; además, es un fuerte factor predisponente para la neumonía asociada con ventilación mecánica.

Tradicionalmente, el tratamiento de la atelectasia en pacientes de la $\mathrm{UCI}$ se ha centrado en la aspiración de secreciones traqueobronquiales complementada, como técnicas de fisioterapia respiratoria y broncoscopia. ${ }^{2}$ Cualquiera que sea la terapia, el objetivo final es reexpandir el área afectada incrementando la presión transpulmonar. Sin embargo, aún en la actualidad no está claro si una modalidad es superior a la otra en el tratamiento de la ALA. La broncoscopia terapéutica ha sido justificada con base en reportes de casos, experiencias 
clínicas anecdóticas, series de casos retrospectivos e informes descriptivos. Las aplicaciones de estas observaciones se han visto obstaculizadas por la heterogeneidad de los pacientes, los mecanismos y extensión de la atelectasia, y las variaciones en las técnicas broncoscópicas. ${ }^{3}$ Esta discrepancia ha llevado a la confusión sobre el papel de la broncoscopia en el tratamiento de esta afección, con notables variaciones en los enfoques entre los médicos. ${ }^{3}$ Tal vez, sobre todo en nuestro medio, la decisión dependa de los recursos con los que se cuenta.

Una de las principales indicaciones de la broncoscopia flexible en la $\mathrm{UCI}$, es precisamente para el tratamiento de las atelectasias. ${ }^{4,5}$ No obstante, el uso de la broncoscopia para las secreciones retenidas y las atelectasias es objeto de fuertes convicciones, pero escasa información. Hay pocas dudas de que la broncoscopia utilizada para la eliminación terapéutica de las secreciones y el lavado pulmonar agresivo es un método seguro, pero su eficacia e impacto en los resultados clínicos sigue sin resolverse. Según algunos autores, cuando se comparó la broncoscopia terapéutica con la higiene bronquial agresiva, hubo poca diferencia en los resultados, y cuando hay presencia de broncograma, ambas estrategias son igualmente malas para resolver la atelectasia. ${ }^{6}$ La broncoscopia y el lavado bronquio-alveolar (LBA) no deberán ser considerados como terapia de primera línea como medida rutinaria en la remoción de secreciones en la UCI. La broncoscopia deberá ser considerada en aquellos casos de ALA, especialmente asociada con alteraciones en la oxigenación si el aspirado rutinario mediante catéter es fallido. ${ }^{6}$

La atelectasia bien establecida a menudo resulta refractaria a una rápida expansión, incluso después de que se haya despejado la vía aérea obstruida. La instrumentación agresiva que ocluye selectivamente las vías respiratorias involucradas y mantiene una alta presión de insuflado regional al grado de exceder las presiones asociadas con una capacidad pulmonar total puede revertir la atelectasia, con un impacto variable en el intercambio gaseoso. No obstante, las indicaciones que exigen medidas tan agresivas y la relativa seguridad de tales maniobras no se han delineado ni verificado de manera convincente. ${ }^{1}$

A continuación, exponemos nuestra experiencia con la insuflación transbroncoscópica en el tratamiento de la ALA refractaria a aspirado bronquial con catéter y fisioterapia respiratoria en pacientes intubados de la UCI.

\section{MATERIAL Y MÉTODOS}

Revisión descriptiva clínico-radiológica retrospectiva de una serie de pacientes graves de nuestra $\mathrm{UCI}$, que cursaron con apoyo ventilatorio mecánico, con vía aérea artificial y una ALA refractaria a aspirado con catéter y fisioterapia respiratoria por un periodo mayor a los cinco días. Usualmente las técnicas de fisioterapia respiratoria en nuestra institución incluyen la hiperinflación manual, inhaloterapia, movilización y cambio de postura del paciente y la percusión torácica una a dos veces por cada turno de 8 horas. Ésta es realizada únicamente por el personal de enfermería en situaciones de ALA. Como ALA, se definió aquella opacidad homogénea extensa correspondiente a varios segmentos o lóbulos pulmonares, con retracción de las estructuras anatómicas adyacentes. Se utilizó un fibrobroncoscopio flexible Olympus LF-TP con canal de trabajo de $2.6 \mathrm{~mm}$ de diámetro. El procedimiento es realizado por uno de los autores y asistido por personal de enfermería, médicos becarios o adscritos a la UCI de nuestro hospital. Posterior a lavado con agua inyectable y aspirado de las secreciones obstructivas localizadas en el o los segmentos correspondientes, el broncoscopio fue enclavado en cada uno de ellos y con una bolsa de Ambu conectada al canal de trabajo por medio de una jeringa de $3 \mathrm{~mL}$ unida a un conector de tubo endotraqueal de $8 \mathrm{~mm}$ de diámetro interno (DI) o directamente con un conector de un tubo endotraqueal pediátrico de $3 \mathrm{~mm}$ de $\mathrm{DI}$, se realizaron de seis a ocho insuflaciones suaves de aire ambiental. Idealmente, dichas insuflaciones no deberían sobrepasar una presión de $30 \circ 10 \mathrm{cmH}_{2} \mathrm{O}$ por arriba de la presión en meseta en pacientes bajo apoyo ventilatorio mecánico y, para ello, un entrenamiento previo utilizando bolsas de Ambu pediátricas nos ayudó a percibir aproximadamente la fuerza manual necesaria para no sobrepasar dichas presiones. ${ }^{7}$ A diferencia de las bolsas de Ambu para adultos, las utilizadas en niños incorporan una válvula de liberación de presión que limita la presión inspiratoria máxima a $40+/-10 \mathrm{cmH}_{2} \mathrm{O}$, lo que reduce el riesgo de barotrauma. Se registró el diagnóstico de la enfermedad de base, el tipo de la vía aérea por donde se insertó el broncoscopio y las radiografías antes y después del procedimiento. Se consideró un procedimiento broncoscópico exitoso aquel con una resolución clínica y radiológica de la ALA en las siguientes horas posterior al procedimiento.

\section{RESULTADOS}

Logramos obtener información de ocho pacientes que cursaron con ALA durante los últimos dos años y que ameritaron tratamiento mediante broncoscopia (Figura 1). En todos los casos no hubo evidencia de broncograma aéreo, lo que indicó un proceso obstructivo proximal a nivel de alguno de los bronquios principales y, consecutivamente, en todos se documentaron grandes tapones obstructivos causados por la presencia de moco espeso y adherente en alguno de los bronquios principales: siete en el bronquio principal izquierdo y sólo uno en el bronquio principal derecho. Siete de los ocho 
procedimientos realizados se consideraron exitosos. No obstante, el pronóstico de vida y complicaciones dependió más de la enfermedad de base que de la ALA en sí y hubo tres fallecimientos, dos de ellos por traumatismo severo (pacientes uno y ocho) y otro por edema cerebral (paciente siete). La mejoría clínica y radiológica se observó inmediatamente posterior al procedimiento en seis pacientes y en otro fue parcial, pero al siguiente día

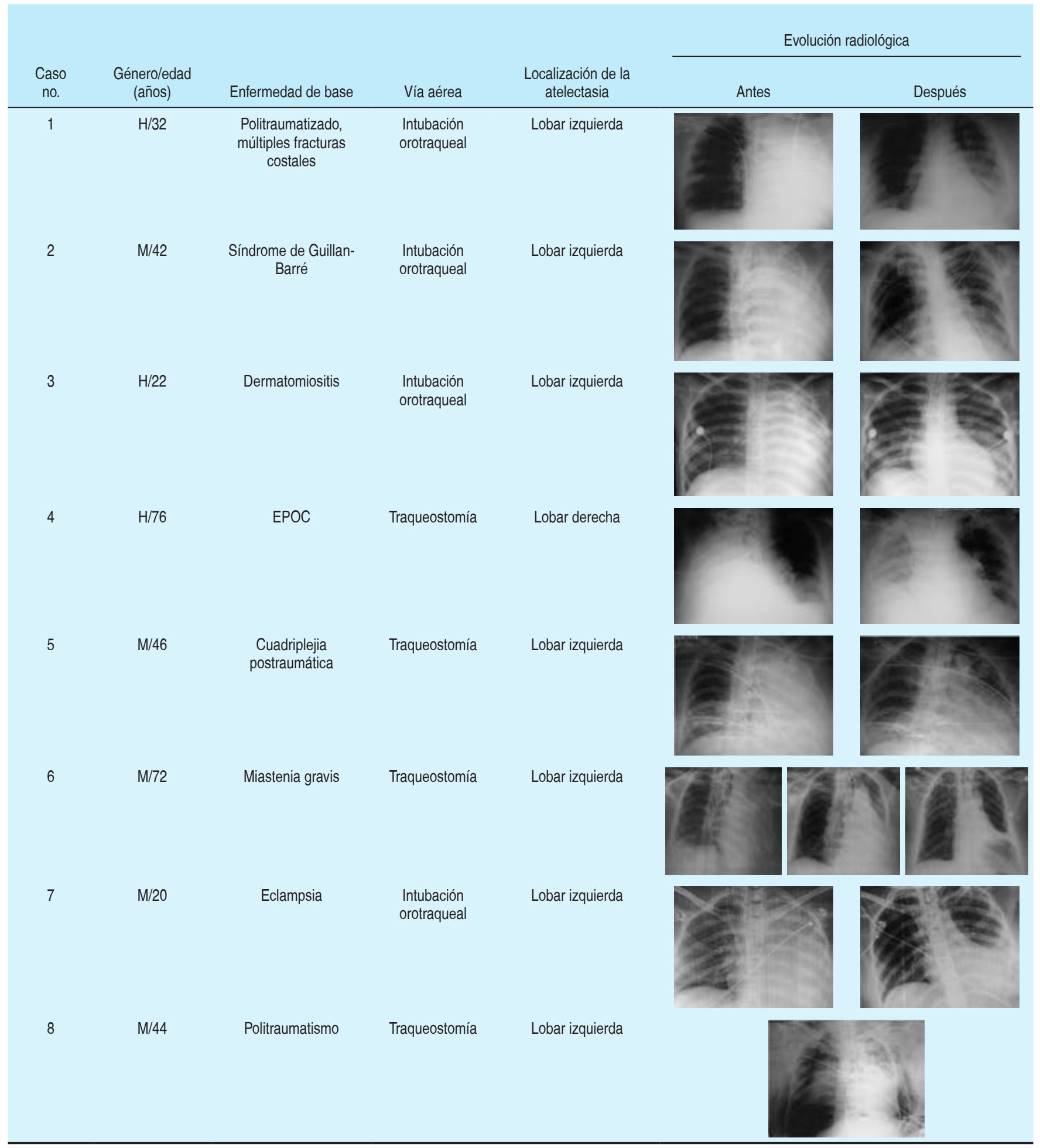

$M=$ mujer $\mathrm{H}=$ hombre; $E P O C$ = enfermedad pulmonar obstructiva crónica.

Figura 1: Características clínicas, cambios radiológicos y resultados de la insuflación pulmonar selectiva con ayuda del broncoscopio flexible. 
hubo una completa resolución de la atelectasia lobar (paciente seis). El paciente ocho presentó como complicación un neumotórax bilateral y enfisema subcutáneo que ameritaron drenaje pleural con tubo. Dicha complicación se atribuyó a que el broncoscopio fue introducido por un orificio de una cánula de traqueostomía muy estrecho, lo que pudo haber impedido la liberación del exceso de presión aplicada durante las insuflaciones manuales con la bolsa de Ambu.

\section{DISCUSIÓN}

Los pacientes bajo ventilación mecánica tienen un riesgo alto de desarrollar atelectasias y es el tubo endotraqueal el responsable de un transporte de moco deteriorado y de una tos ineficaz, debido a la falta del cierre glótico adecuado. La ALA, en la mayoría de los casos, puede ser resuelta con una fisioterapia respiratoria convencional bien aplicada y su resolución usualmente es observada en las siguientes 24 a 48 horas. ${ }^{8}$ El manejo rápido de la atelectasia puede ser esencial para evitar complicaciones como la hipoxemia secundaria a cortocircuitos, neumonía e insuficiencia respiratoria. ${ }^{3}$ Después de este periodo, de persistir o empeorar la ALA, deberá considerarse la broncoscopia. Se ha demostrado que la broncoscopia es segura y útil en pacientes con atelectasia, con tasas de éxito medidas objetivamente por un mejor intercambio de gases o una mejor aireación radiográfica que varía de 19 a $89 \%,{ }^{3}$ una mortalidad menor a $0.5 \%$ y una tasa de complicaciones de 0.08 a $5 \% .{ }^{8}$ Además, la fisioterapia respiratoria tiene sus limitaciones en pacientes traumatizados, hemodinámicamente inestables, con quemaduras extensas u obesidad mórbida. La movilización del paciente en su cama puede representar un riesgo para una extubación accidental. Idealmente se requiere personal capacitado disponible las 24 horas y de realizarse por enfermería, representaría una carga significativa de trabajo. ${ }^{9} \mathrm{En}$ efecto, se puede argumentar que las enfermeras son capaces de proporcionar fisioterapia a sus pacientes y pueden reemplazar a los fisioterapeutas en su ausen- cia. Aunque esto es cierto, es prácticamente imposible que una enfermera brinde fisioterapia adecuada en las $\mathrm{UCI}$ con una alta tasa de rotación de pacientes, una baja relación enfermera-paciente y la falta de capacitación adecuada en principios básicos de fisioterapia. ${ }^{9}$

El mayor beneficio y ventaja de un abordaje broncoscópico en pacientes con un colapso pulmonar completo, o casi-completo, es la posibilidad de inspeccionar directamente las vías respiratorias y la opción de obtener muestras de líquidos o tejidos para un diagnóstico definitivo, reservando el manejo conservador para pacientes con atelectasias segmentarias o subsegmentarias ${ }^{10}$ (Figura 2). La broncoscopia para el tratamiento de la ALA es beneficiosa en la mayoría de los pacientes de la $\mathrm{UCI}$ al mejorar el intercambio de gases y las propiedades mecánicas del sistema respiratorio, con efectos positivos que duran hasta 24 horas. ${ }^{11}$

Mientras un tapón de moco puede causar una ALA, su aspirado puede no ser suficiente para resolver la atelectasia y existe un subgrupo de pacientes en quienes la simple aspiración bronquial a través del broncoscopio podría no ser mejor que una terapia más conservadora, debido a que no conlleva un reclutamiento de los alveolos colapsados. De aquí se desprende por qué algunos autores han defendido el uso de la insuflación, además de la broncoscopia estándar para el tratamiento de la atelectasia. ${ }^{2}$ La insuflación no selectiva por medio del tubo endotraqueal, de la misma manera que otras maniobras de reclutamiento alveolar, podría someter las áreas de pulmón no atelectasiado a presiones significativamente mayores y en riesgo de barotrauma o, incluso, comprometer el gasto cardiaco. ${ }^{12,13}$ Además, se requiere una presión transpulmonar relativamente más elevada para abrir las áreas poco distensibles de pulmón atelectasiado, de forma similar a lo que sucede al intentar inflar un globo. Con base en ello es como se ha sugerido la introducción de ventilación a presión positiva directamente en el área de pulmón colapsado, para superar la presión crítica de apertura de la vía aérea sin afectar las áreas de baja resistencia y mayor distensibilidad del pulmón normal adyacente con la posibilidad de ser sobredistendidas.
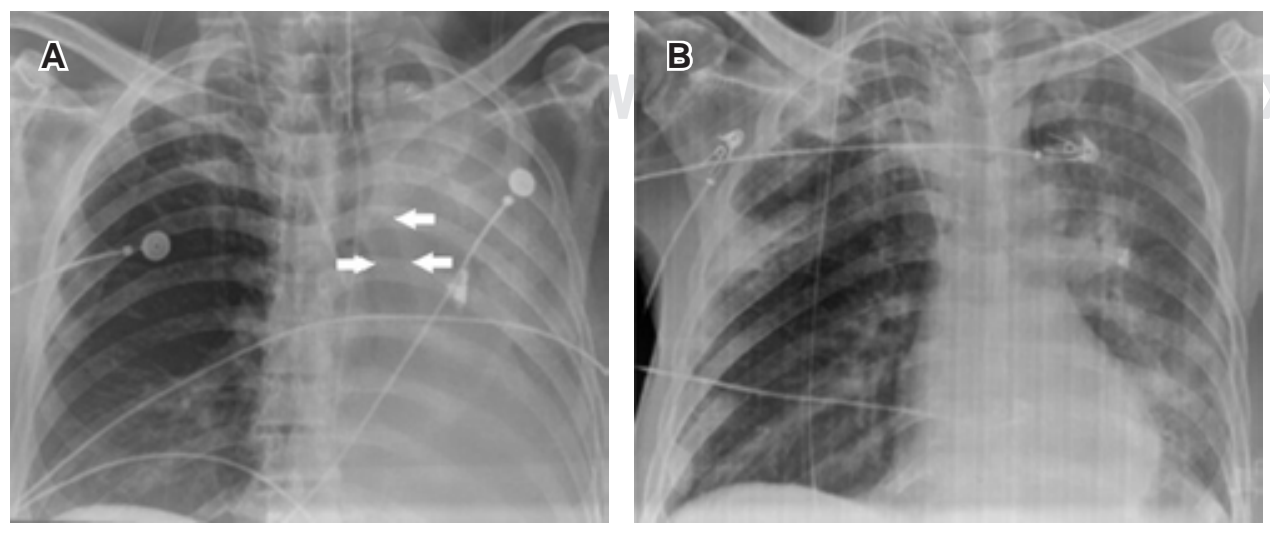

Figura 2:

Radiografías de tórax de un hombre de 54 años con traumatismo craneoencefálico severo y hemorragia subaracnoidea bajo apoyo ventilatorio mecánico vía tubo endotraqueal. A) Se evidenció una atelectasia lobar izquierda con broncograma aéreo (flechas blancas) de más de siete días de evolución. B) Finalmente, evolucionó satisfactoriamente con fisioterapia respiratoria; no obstante, se complicó con una neumonía asociada al ventilador. 
Inicialmente, en las técnicas para insuflar de manera selectiva el pulmón atelectasiado, se utilizó un broncoscopio rígido o flexible con balón oclusivo o, por medio de éste, un catéter con balón distal para insuflar emboladas de aire con jeringa con buenos resultados. ${ }^{14-18}$ Posteriormente, mediante el simple enclavamiento bronquial del broncoscopio flexible e insuflaciones con una bolsa de Ambu por el canal de trabajo, se obtuvieron resultados con una tasa de éxito de $70-93 \%$. $^{19-22}$ La presión ejercida por dichas insuflaciones fue monitoreada cuidando no superar los $30 \mathrm{cmH}_{2} \mathrm{O}$ y, como parte del mecanismo de seguridad para evitar el daño por sobredistensión alveolar, se ha argumentado que el enclavamiento bronquial del broncoscopio suele ser imperfecto, lo que permite la fuga de presión del aire si ésta fuera excesiva.

La insuflación selectiva es reservada para aquellos casos de ALA refractarios a tratamientos convencionales, por lo regular ya con una duración de más de cinco días. Todos nuestros casos correspondieron a ALA de más de ese periodo de tiempo y el pulmón izquierdo fue el más afectado, lo cual es explicable por la diferencia anatómica entre el bronquio principal derecho y el izquierdo, cuya posición más horizontal lo hace de difícil acceso para los catéteres de aspiración de secreciones. La mejoría clínica y radiológica fue observada al poco tiempo de realizada la maniobra de insuflación en la mayoría de nuestros pacientes y sólo en los casos cinco, seis y siete, observamos la mejoría en una forma más gradual y, probablemente, como lo mencionan Susini y colaboradores, ${ }^{18}$ después de una reexpansión parcial inicial puede observarse una mejoría espontánea adicional 24 horas más tarde, debido a que la reexpansión incompleta en sí puede promover un reclutamiento alveolar adicional en las áreas menos distensibles. En los tres casos mencionados, el lóbulo inferior izquierdo fue más difícil de expandir, tal vez porque durante la posición totalmente supina, el peso del corazón y el mediastino estrecha y pellizca los bronquios del lóbulo inferior izquierdo. ${ }^{1}$ Nuestra sugerencia sería realizar un mayor número de insuflaciones en dicho lóbulo.

Ya desde 1939, Macklin había postulado que un requisito básico para la ruptura alveolar es la existencia de un gradiente de presión entre el alvéolo y el tejido periférico. ${ }^{23}$ En relación con el caso número ocho, el neumotórax y enfisema subcutáneo como una complicación de la insuflación transbroncoscópica, ha sido descrita sobre todo en niños y atribuida a que el diámetro del broncoscopio compromete el calibre de la vía aérea al disminuir su área transversal y ocasionar un mecanismo de válvula al impedir la liberación de presión ejercida en la vía aérea durante una maniobra de insuflación. Por ejemplo, un broncoscopio con un diámetro de $5.7 \mathrm{~mm}$ introducido en un tubo endotraqueal de $8.0 \mathrm{~mm}$ de diámetro interno reduce su área trans- versal en $66 \%$, lo que podría ocasionar presiones inspiratorias tan altas como los $60 \mathrm{cmH}_{2} \mathrm{O} .^{2}$ Para evitar el barotrauma, el endoscopista deberá confirmar que existe suficiente espacio alrededor del broncoscopio dentro del tubo endotraqueal o la cánula de traqueostomía y desinflar el globo, y con ello permitir la liberación de presión que podría ser inadvertidamente excesiva durante la maniobra de insuflación. ${ }^{24-26}$

\section{CONCLUSIONES}

La insuflación pulmonar selectiva parece ser un método efectivo y seguro para el tratamiento de la ALA refractaria al aspirado traqueobronquial y fisioterapia respiratoria en pacientes bajo apoyo ventilatorio mecánico; no obstante, como cualquier técnica broncoscópica, los resultados son dependientes de la experiencia del operador. Durante las insuflaciones son preferibles las variaciones de presión (presión crítica de apertura) que de volumen de aire. Durante el procedimiento, siempre deberán ser considerados los mecanismos auxiliares de liberación de presión como son el enclavamiento imperfecto del broncoscopio en los bronquios segmentarios, un tubo endotraqueal o cánula de traqueostomía con un diámetro adecuado y/o desinflar temporalmente el globo oclusor distal.

\section{REFERENCIAS}

1. Marini JJ. Acute lobar atelectasis. Chest. 2019;155(5):10491058.

2. Kreider ME, Lipson DA. Bronchoscopy for atelectasis in the ICU: a case report and review of the literature. Chest. 2003;124(1):344-350.

3. Surka A, Bowling MR, Chin R, Haponik E, Conforti J. Bronchoscopic myths and legends: bronchoscopy in the treatment of lobar atelectasis. Clin Pulm Med. 2007;14:302305.

4. Alvarez-Maldonado $P$, Núñez-Pérez-Redondo F, CasillasEnriquez JD, Navarro-Reynoso F, Cisero-Sabido R. Indications and efficacy of fiberoptic bronchoscopy in the ICU: have they changed since its introduction in clinical practice? ISRN Endoscopy. 2013; Article ID 217505, 6 pages.

5. Estella A. Analysis of 208 flexible bronchoscopies performed in an intensive care unit. Med Intensiva. 2012;36(6):396-401.

6. Garden JA. Bronchoscopy in the intensive care unit. In: Ernst A. Introduction to bronchoscopy. NY, USA: Cambridge University Press; 2009. p. 120.

7. Flores-Franco RA, Centeno-Hernandez $\mathrm{H}$. Insufflation of collapsed lung using the flexible bronchoscope. J Bronchol. 2006;13:46.

8. Raoof S. Controversy. Is bronchoscopy indicated in the management of atelectasis? Con: bronchoscopy. J Bronchol. 2002;9:52-58.

9. Mehrishi S. Controversy. Is bronchoscopy indicated in the management of atelectasis? Pro: bronchoscopy. J Bronchol. 2002;9:46-51.

10. Toolsie OG, Adrish M, Zaidi SAA, Diaz-Fuentes G. Comparative outcomes of inpatients with lung collapse managed by bronchoscopic or conservative means. BMJ Open Respir Res. 2019;6(1):e000427.

11. Smeijsters KMG, Bijkerk RM, Daniels JMA, van de Ven PM, Girbes ARJ, Heunks LMA, et al. Effect of bronchoscopy on gas exchange and respiratory mechanics in critically ill patients 
with atelectasis: an observational cohort study. Front Med (Lausanne). 2018;5:301.

12. Okamoto T, Niikawa $\mathrm{H}$, Wheeler $\mathrm{D}$, Ayyat KS, Basem S, Itoda $Y$, et al. Selective recruitment of large lower lobe atelectasis on donor back table in rejected donor lungs. Transplant Direct. 2019;5(5):e453.

13. Hansen LK, Sloth E, Nielsen J, Koefoed-Nielsen J, Lambert $\mathrm{P}$, Lunde $\mathrm{S}$, et al. Selective recruitment maneuvers for lobar atelectasis: effects on lung function and central hemodynamics: an experimental study in pigs. Anesth Analg. 2006;102(5):1504-1510.

14. Bowen TE, Fishback ME, Green DC. Treatment of refractory atelectasis. Ann Thorac Surg. 1974;18(6):584-589.

15. Lee TS, Wright BD. Selective insufflation of collapsed lung with fiberoptic bronchoscope and Swan-Ganz catheter. Intensive Care Med. 1981;7(5):241-243.

16. Millen JE, Vandree J, Glauser FL. Fiberoptic bronchoscopic balloon occlusion and reexpansion of refractory unilateral atelectasis. Crit Care Med. 1978;6(1):50-55.

17. Harada K, Mutsuda T, Saoyama N, Taniki T, Kimura H. Reexpansion of refractory atelectasis using a bronchofiberscope with a balloon cuff. Chest. 1983;84(6):725-728.

18. Susini G, Sisillo E, Bortone F, Salvi L, Moruzzi P. Postoperative atelectasis reexpansion by selective insufflation through a balloon-tipped catheter. Chest. 1992;102(6):1693-1696.

19. Tsao TC, Tsai YH, Lan RS, Shieh WB, Lee CH. Treatment for collapsed lung in critically ill patients. Selective intrabronchial air insufflation using the fiberoptic bronchoscope. Chest. 1990;97(2):435-438.

20. Haenel JB, Moore FA, Moore EE, Read RA. Efficacy of selective intrabronchial air insufflation in acute lobar collapse. Am J Surg. 1992;164(5):501-505.
21. van Heerden PV, Jacob W, Cameron PD, Webb S Bronchoscopic insufflation of room air for the treatment of lobar atelectasis in mechanically ventilated patients. Anaesth Intensive Care. 1995;23(2):175-177.

22. Wohlauer MV, Moore EE, Haenel JB, Burlew CC, Barnett CC Jr. Selective intrabronchial air insufflation for acute lobar collapse in the surgical Intensive Care Unit. J Surg Radiol. 2011;2(2):178180.

23. Macklin CC. Transport of air along sheaths of pulmonic blood vessels from alveoli to mediastinum: Clinical implications. Arch Intern Med. 1939;64:913-926.

24. Mirza M, Baram D. Bilateral pneumothoraces complicating tracheal insufflation in a nonintubated adult. J Bronchol. 2008;15:173-175.

25. Iannoli ED, Litman RS. Tension pneumothorax during flexible fiberoptic bronchoscopy in a newborn. Anesth Analg. 2002;94(3):512-513.

26. Scolieri P, Adappa ND, Coticchia JM. Value of rigid bronchoscopy in the management of critically ill children with acute lung collapse. Pediatr Emerg Care. 2004;20(6):384-386.

\section{Correspondencia:}

René Agustín Flores-Franco

Hospital General Regional

«Dr. Salvador Zubirán Anchondo»,

Av. Cristóbal Colón y Teófilo Borunda Núm. 510,

Col. Centro, 31000, Chihuahua, Chih., México.

E-mail: rflores99@prontomail.com 